\title{
Improvement of the inhibitory effect of xanthones on NO production by encapsulation in PLGA nanocapsules
}

\author{
MARIBEL TEIXEIRA ${ }^{1,2}$, FÁTIMA CERQUEIRA ${ }^{1}$, CARLOS MAURÍCIO BARBOSA $^{3,4}$, \\ MARIA SÃO JOSÉ NASCIMENTO ${ }^{1,5}, \&$ MADALENA PINTO ${ }^{1,6}$
}

${ }^{1}$ CEQOFFUP_Centro de Estudos de Química Orgânica, Fitoquímica e Farmacologia da Universidade do Porto, Faculdade de Farmácia, Universidade do Porto, R. Aníbal Cunha, 164, 4050-047 Porto, Portugal, ${ }^{2}$ Instituto Superior de Ciências da SaúdeNorte, R. Central de Gandra, 1317, 4585-116 Gandra, Paredes, Portugal, ${ }^{3}$ CTMUP_Serviço de Tecnologia Farmacêutica, Faculdade de Farmácia, Universidade do Porto, R. Aníbal Cunha, 164, 4050-047 Porto, Portugal, ${ }^{4}$ CETMED—Centro Tecnológico do Medicamento, R. do Passeio Alegre, 840, 4150-574 Porto, Portugal, ${ }^{5}$ Serviço de Microbiologia, Faculdade de Farmácia, Universidade do Porto, R. Aníbal Cunha, 164, 4050-047 Porto, Portugal, and ${ }^{6}$ Serviço de Química Orgânica, Faculdade de Farmácia, Universidade do Porto, R. Aníbal Cunha, 164, 4050-047 Porto, Portugal

\begin{abstract}
For the first time the inhibitory effect of xanthone and 3-methoxyxanthone on nitric oxide (NO) production by IFN- $\gamma / \mathrm{LPS}$ activated J774 macrophage cell line is reported. A remarkable improvement of this effect promoted by encapsulation of these compounds in nanocapsules of poly (DL-lactide-co-glycolide) (PLGA) is also demonstrated. A weak inhibitory effect of 3.6\% on NO production by activated macrophages was observed for xanthone at the highest studied concentration (100 $\mu \mathrm{M})$. This effect was slightly higher for 3-methoxyxanthone at the same concentration, producing a reduction of $16.5 \%$ on NO production. In contrast, equivalent concentrations of xanthone and 3-methoxyxanthone incorporated in nanocapsules produced a significant decrease on NO production of 91.8 and $80.0 \%$, respectively. Empty nanocapsules also exhibited a slight NO inhibitory activity, which may be due to the presence of soybean lecithin in the composition of the nanosystems. The viability of the macrophages was not affected either by free or nanoencapsulated xanthones. Fluorescence microscopy analysis confirmed that a phagocytic process was involved in the macrophage uptake of xanthone- and 3-methoxyxanthoneloaded PLGA nanocapsules. Phagocytosis might be the main mechanism responsible for the enhancement of the intracellular delivery of both compounds and consequently for the improvement of their biological effect.
\end{abstract}

Keywords: Xanthone, 3-methoxyxanthone, nanocapsules, PLGA, nitric oxide, 7774 macrophages

\section{Introduction}

Nitric oxide (NO) is a gas produced by constitutive and inducible isoforms of NO synthase (NOS) from L-arginine and molecular oxygen in a variety of cells (Gross and Wollin 1995, Aramaki 2000). Constitutive NOS isoforms are expressed in endothelial and neuronal cells and are calcium- and calmudolindependent (Kolb and Kolb-Bachofen 1992, Aramaki 2000). NO produced by these NOS acts as an intracellular messenger. In contrast, the inducible NOS isoform (iNOS) is not dependent on calcium or calmudolin and its expression is induced by immunological stimuli in virtually all nucleated mammalian cells (Gross and Wollin 1995). Activation of macrophages with some cytokines and/or with lipopolysaccharide (LPS) induces the production of large quantities of NO generated by iNOS (Lowenstein et al. 1993, Gross and Wollin 1995). NO regulates several important physiological processes, namely the maintenance of normal blood pressure, neuronal mediation and inflammatory responses and also acts as a defensive agent by damaging pathogenic DNA (Kuo and Schoeder 1995). However, excessive and unregulated NO synthesis has been implicated as causal or contributing to

Correspondence: M. Pinto, CEQOFFUP—Centro de Estudos de Química Orgânica, Fitoquímica e Farmacologia da Universidade do Porto, Faculdade de Farmácia, Universidade do Porto, R. Aníbal Cunha, 164, 4050-047, Porto, Portugal. Tel: 351222057358. Fax: 351222003 977. E-mail: madalena@ff.up.pt 
pathophysiological conditions, including many lethal and debilitating diseases of humans, such as vascular shock, stroke, diabetes, arthritis and chronic inflammation (Gross and Wollin 1995).

Xanthones ( $=9 H$-xanthen-9-ones $)$ are heterocyclic compounds based on the dibenzo- $\gamma$-pirone nucleus (Figure 1) including natural, semi synthetic and totally synthetic structures. Xanthones have extensive biological activities such as hepatoprotection, antiinflammation, immunomodulation, growth inhibition of human cancer cell lines and modulation of protein kinase C (PKC) (Fernandes et al. 1995, Lin et al. 1996, Pinto and Nascimento 1997, Gonzalez et al. 1999, Peres et al. 2000, Pedro et al. 2002, Saraiva et al. 2002a,b, Pinto and Sousa 2003, Saraiva et al. 2003). Nevertheless, studies on reduction of NO production by xanthone derivatives are scarce. Some data are reported only for mangiferin, which is a C-glucosylxanthone (García et al. 2002, Leiro et al. 2003).

Xanthone and 3-methoxyxanthone (Figure 1) were already evaluated in our group concerning different biological activities, namely inhibitory activity of monoamine oxidase (Thull et al. 1993), immunomodulatory (Pinto and Nascimento 1997), PKC modulation (Saraiva et al. 2002b) and growth inhibitory activity of human cancer cell lines (Pedro et al. 2002). In the present work, the inhibitory effect of these xanthones on the production of NO by the murine macrophage cell line J774 is reported for the first time. In order to enhance the intracellular delivery of the xanthones and thus to improve their biological activity, both compounds were encapsulated in biodegradable nanocapsules of poly (DL-lactide-coglycolide) (PLGA). In fact, nanoencapsulation is known to increase the intracellular delivery of several drugs into phagocytic cells (Morin et al. 1994, Wang and Zhang 2001). Nanocapsules can be easily obtained by interfacial deposition of the polymer around an oily core in which lipid-soluble drugs can be dissolved, as we have described previously (Teixeira et al. 2004). PLGA has been selected for nanocapsule preparation, since polyesters, including poly (lactic acid), poly (glycolic acid) and their copolymers, have emerged as the most widely studied class of polymers for pharmaceutical use due to their biocompatibility and biodegradability characteristics (Jain et al. 1998).



$\mathrm{R}=\mathrm{I}$ : Xanthone

$\mathrm{R}=\mathrm{OCH}_{3}$ : 3-Methoxyxanthone

Figure 1. Chemical structure of xanthones.
The main goal of the present study was to evaluate in vitro the inhibitory effect of xanthone and 3methoxyxanthone on NO production by the activated macrophage cell line J774 as well as to improve that effect through the encapsulation of the compounds in PLGA nanosystems.

\section{Materials and methods}

\section{Chemicals and reagents}

Xanthone, PLGA (50:50) MW 50,000-75,000, Pluronic F-68 and soybean lecithin (40\% purity by thin-layer chromatography) were purchased from Sigma Chemical Co. (St Louis, USA). 3-Methoxyxanthone was synthesized in our laboratory by alkaline cyclization of 2-hydroxy-2',4-dimethoxybenzophenone as previously described (Fernandes et al. 1998). Myritol 318 (caprilic/capric acid triglyceride) was kindly supplied by Henkel (Lisbon, Portugal). Foetal bovine serum (FBS) and RPMI-1640 were purchased from Gibco Invitrogen Co. (Barcelona, Spain). Other chemicals were of analytical grade and unless otherwise indicated were purchased from Sigma.

\section{Preparation and characterization of nanocapsules}

Nanocapsules containing either xanthone or 3-methoxyxanthone were prepared as previously described (Teixeira et al. 2004). Briefly, about $50 \mathrm{mg}$ of PLGA and $100 \mathrm{mg}$ of soybean lecithin were dissolved in $10 \mathrm{ml}$ of acetone. Xanthone $(7.2 \mathrm{mg})$ or 3-methoxyxanthone $(16.8 \mathrm{mg}$ ) was dissolved in $0.6 \mathrm{ml}$ of Myritol 318 and the obtained solution was added to the acetonic solution. The final solution was poured into $20 \mathrm{ml}$ of an aqueous solution of Pluronic F- $680.5 \%$ (w/v) under moderate stirring, leading to the formation of nanocapsules. Acetone was then removed under vacuum and the colloidal dispersion of nanocapsules was concentrated to $5 \mathrm{ml}$ by evaporation under reduced pressure. Non-encapsulated xanthones (either xanthone or 3-methoxyxanthone) were separated by ultrafiltration/centrifugation (centrifugal filter devices Centricon YM-50, Millipore ${ }^{\circledR}$, Bedford, USA) at $4000 \mathrm{~g}$ for $2 \mathrm{~h}$ (Beckman UL-80 ultracentrifuge, Albertville, USA).

Empty nanocapsules were prepared according to the same procedure but omitting xanthones in the organic phase. Nanoemulsion (NE) was prepared in the same way as nanocapsules, but omitting the polymer and the xanthones. Nanospheres (NS) were obtained omitting lecithin, Myritol 318 and xanthones. Lecithin dispersion (LD) was prepared omitting polymer, Myritol 318 and xanthones. Myritol 318 aqueous dispersion (MD) was obtained omitting polymer, lecithin and xanthones. Freshly prepared formulations were used in all experiments. 
Mean particle size of xanthone- and 3-methoxyxanthone-loaded nanocapsules and empty nanocapsules, determined by photon correlation spectroscopy (Zetasizer 5000, Malvern Instruments, Malvern, UK) was $273 \pm 18,271 \pm 16$ and $261 \pm 17 \mathrm{~nm}$, respectively.

The amount of xanthone and 3-methoxyxanthone in nanocapsules was determined following dissolution of aliquots of nanocapsule dispersions in acetonitrile by a previously validated HPLC method (Teixeira et al. 2003). Incorporation efficiency was higher than $77 \%$ for nanocapsule formulations containing either xanthone or 3-methoxyxanthone.

\section{NO production assay}

The murine J774 macrophage cell line was maintained in RPMI-1640 medium supplemented with $10 \%$ heatinactivated FBS serum, $2 \mathrm{mM}$ glutamine and $50 \mu \mathrm{g} / \mathrm{ml}$ of gentamicin (designated thereafter as culture medium) at $37^{\circ} \mathrm{C}$ in a $5 \% \mathrm{CO}_{2}$ humidified atmosphere. Cells were added to 96 -well flat-bottom microplates at a density of $0.5 \times 10^{6}$ cells $/ \mathrm{ml}$ and allowed to adhere for $2 \mathrm{~h}$. To induce iNOS, culture medium was replaced by fresh medium containing LPS $(1 \mu \mathrm{g} / \mathrm{ml})$ and IFN- $\gamma(100 \mathrm{U} / \mathrm{ml})$. Aqueous dispersions of nanocapsules containing xanthone or 3-methoxyxanthone as well as solutions of xanthone or 3-methoxyxanthone in DMSO were diluted with culture medium (concentrations ranging from 2 to $100 \mu \mathrm{M}$ ) and tested. Equivalent concentrations of empty nanocapsules were also tested. Final concentrations of dimethylsulfoxide (DMSO) showed no interference with the tested biological activity. Sample dilutions were added together with stimulus and their effect on NO production was evaluated after $24 \mathrm{~h}$ of incubation at $37^{\circ} \mathrm{C}$, quantifying nitrite accumulation in cell culture supernatant by the Griess reaction (Green et al. 1982).

\section{NO scavenging assay}

Sodium nitroprusside $(5 \mathrm{mM})$ in PBS was mixed with the different samples (xanthone- and 3-methoxyxanthone-loaded nanocapsules, empty nanocapsules as well as xanthone and 3-methoxyxanthone solutions in DMSO) and with ethanol/PBS (1:9) and incubated for $150 \mathrm{~min}$ at $25^{\circ} \mathrm{C}$ in 96-well flat-bottom microplates. After incubation, Griess reagent $(1 \% \mathrm{w} / \mathrm{v}$ sulphanilamide and $0.1 \% \mathrm{w} / \mathrm{v}$ naphtylethylenediamide in $5 \% \mathrm{v} / \mathrm{v}$ phosphoric acid) was added and allowed to react during $10 \mathrm{~min}$ at $25^{\circ} \mathrm{C}$ and the absorbance was measured at $550 \mathrm{~nm}$. Controls consisted of ethanol/ PBS (for $0 \%$ of nitrite production) and sodium nitroprusside (for $100 \%$ of nitrite production). The eventual interference of the samples with the Griess reagent was also evaluated, using the same procedure but omitting the sodium nitroprusside. Scavenging activity, determined in terms of percentage of nitrite formation, was present when the percentage of nitrite formed in the presence of the sample was less than $70 \%$ of the sodium nitroprusside control (De las Heras 1997).

\section{Macrophage viability}

To evaluate the eventual toxicity of both xanthones and the respective nanocapsule formulations on J774 macrophages the MTT-assay was used (Mossman 1983). This assay measures the ability of viable cells to reduce the tetrazolium salt of 3-(4,5dimethylthiazol-2-yl)-2,5-diphenyltetrazoium bromide (MTT) to formazan. Briefly, macrophages plated in 96-well flat-bottom microplates were activated with IFN- $\gamma /$ LPS and exposed for $24 \mathrm{~h}$ to serial concentrations of the different samples. Following this incubation period, the MTT solution $(1 \mathrm{mg} / \mathrm{ml})$ was added. After $4 \mathrm{~h}$ of incubation, formazan was solubilized overnight at $37^{\circ} \mathrm{C}$ in a sodium lauryl sulfate/ $N, N$-dimethylformamide (SDS/DMF) solution $(20 \%$ SDS in a $50 \%$ solution of DMF, $\mathrm{pH}$ 4.7). Absorbance of the coloured solution was measured at $550 \mathrm{~nm}$. Results were expressed, as percentage of viable cells compared to control cells incubated with fresh culture medium $(100 \%$ viability). Cell toxicity was considered when the viability of the exposed cells was less than $70 \%$.

\section{Uptake of nanocapsules by $\mathfrak{7 7 7 4}$ macrophage cell line}

The fluorescence exhibited by xanthone and 3-methoxyxanthone was used to study the cellular uptake of the respective nanosystems by fluorescence microscopy. Macrophages were plated at a density of $0.5 \times 10^{6}$ cells $/ \mathrm{ml}$ onto glass cover slips in 6 -well plates and allowed to adhere for $2 \mathrm{~h}$. Then macrophages were activated with IFN- $\gamma / \mathrm{LPS}$, as described above and incubated with $100 \mu \mathrm{M}$ of each nanocapsule formulation for $4 \mathrm{~h}$ at $4^{\circ} \mathrm{C}$ or at $37^{\circ} \mathrm{C}$. After incubation, cells were washed three times with PBS and fixed with $4 \%$ paraformaldehyde for $10 \mathrm{~min}$ at room temperature. Cells were observed with an Axiovert 200M fluorescence microscope (Carl Zeiss, Germany) equipped with a filter set standard for DAPI. The images were acquired with an Axiocam Camera (Carl Zeiss, Germany) at different focus positions with intervals of $0.5 \mathrm{~mm}$ and deconvolved with Axiovision LE 4.1 (Carl Zeiss, Germany).

\section{Statistics}

Results are presented as mean values \pm SEM of at least three experiments. Paired $t$-test was used to evaluate the statistical significance of differences. 
(a) - Basal production
口 $100 \%$ production
$\mathrm{XAH}$
$\mathrm{XAH}$ Hes

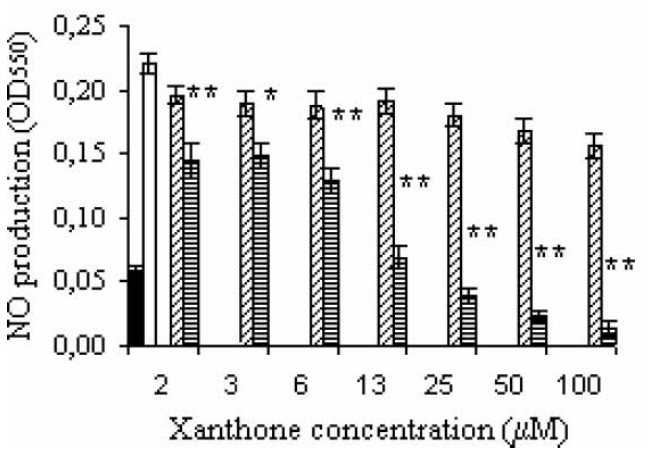

(b)

Basal production 口 $100 \%$ production G Empty Hes 目XAH Hes

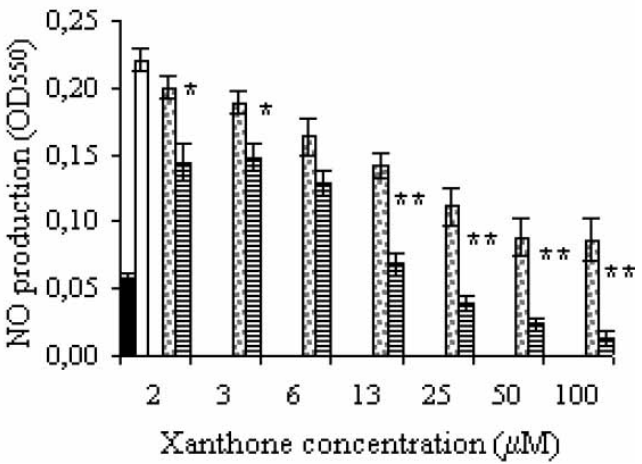

Figure 2. Effect of xanthone (XAN) and xanthone-loaded nanocapsules (XAN Ncs) (2a) and of empty nanocapsules (Empty Ncs) and xanthone-loaded nanocapsules (XAN Ncs) $(2 \mathrm{~b})$ on NO production by IFN- $\gamma / \mathrm{LPS}$ stimulated J774 macrophages. Results show mean values $\pm \operatorname{SEM}(n=5) . \star P<0.05, \star \star P<0.01$

$P$ values $<0.05$ or $<0.01$ were considered statistically significant. Statistic calculations were performed with SPSS for Windows (Release 11.5).

\section{Results and discussion}

\section{Effect of free and encapsulated xanthones on NO} production

The effect of xanthone, 3-methoxyxanthone and the respective nanocapsule formulations on NO production by the IFN- $\gamma /$ LPS activated murine macrophage cell line J774 was evaluated (Figures 2 and 3). As shown in Figure 2a, NO production was weakly affected by the presence of free xanthone. In fact, even at the highest studied concentration $(100 \mu \mathrm{M})$ the inhibition of NO production was only $3.6 \%$. In contrast, an equivalent concentration of xanthone incorporated in nanocapsules produced a significant decrease on NO production (91.8\%; $P<0.01$ ). Results also showed that for all tested concentrations, xanthone-loaded nanocapsules were always associated with a significant decrease $(P<0.05)$ of NO production when compared with free xanthone.

Comparing Figures 2a and 3a, 3-methoxyxanthone showed a higher inhibitory effect on NO production than xanthone. In fact, a $100 \mu \mathrm{M}$ concentration of 3-methoxyxanthone caused a $16.5 \%$ inhibition of NO production. Moreover, the incorporation in nanocapsules of an equivalent concentration of 3-methoxyxanthone caused a decrease of $80 \%$, showing that, as it was observed for xanthone, nanoencapsulation of this compound also leads to a significant $(P<0.01)$ improvement of its NO inhibitory effect.
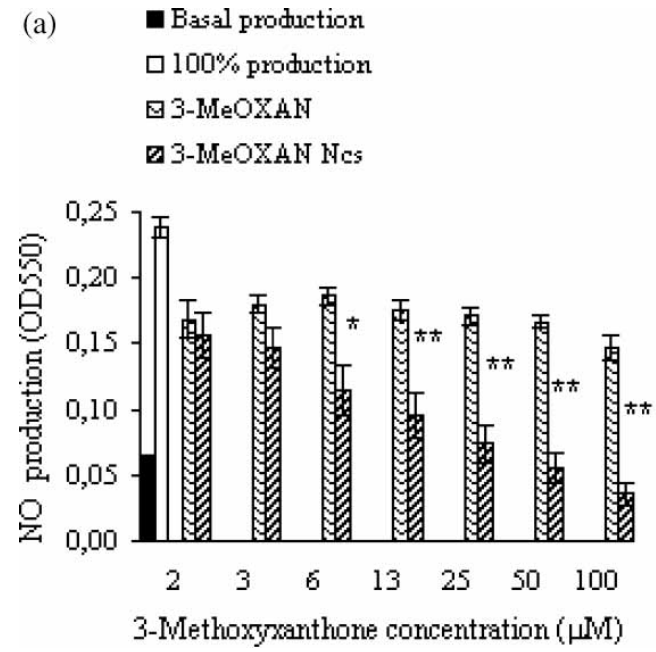

(b)
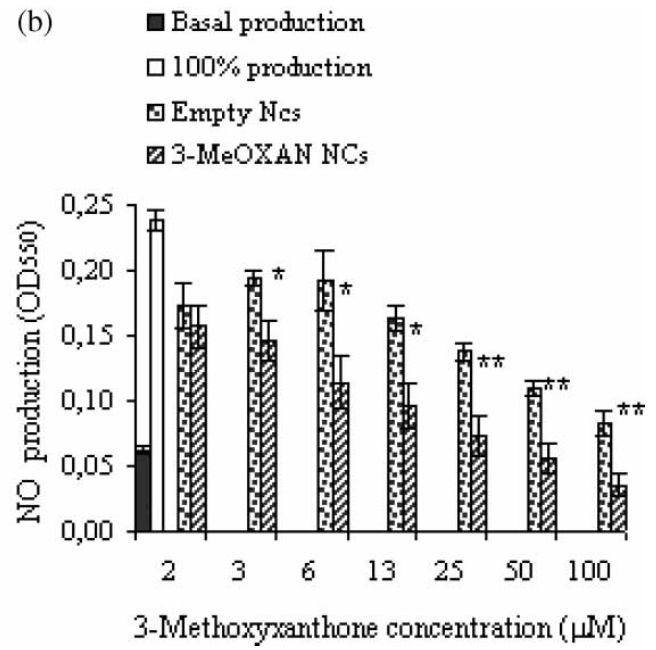

Figure 3. Effect of 3-methoxyxanthone (3-MeOXAN) and 3-methoxyxanthone-loaded nanocapsules (3-MeOXAN Ncs) (3a) and of empty nanocapsules (Empty Ncs) and 3-methoxyxanthone-loaded nanocapsules (3-MeOXAN Ncs) (3b) on NO production by IFN- $\gamma / \mathrm{LPS}$ stimulated J774 macrophages. Results show mean values $\pm \operatorname{SEM}(n=5) . \star P<0.05, \star \star P<0.01$. 
Results from Figures $2 b$ and $3 b$ show that empty nanocapsules also produced an inhibitory effect on NO production. Nevertheless, the comparison of the inhibitory activities of empty and xanthones-loaded nanocapsules showed that these latter were always associated with a significantly higher NO inhibitory effect $(P<0.05)$. Furthermore, the difference between the effects of nanocapsules containing xanthones and empty nanocapsules was significantly higher $(P<0.05)$ than the effect of free xanthones. Thus, an additive effect due to the presence of the xanthones was accompanied by an effect due to the nanoencapsulation of the compounds.

NO inhibitory activity detected for all tested products was not due to a toxic effect, since the J774 activated macrophages exposed to the different samples always showed cell viability higher than $70 \%$. A direct scavenging activity of NO either by the tested xanthonic compounds or by the nanocapsule formulations was also excluded since no NO scavenging activity was observed (data not shown).

In order to investigate which nanocapsule excipients were responsible for the NO inhibitory effect produced by empty nanocapsules, the following formulations were prepared: nanoemulsion (NE), nanospheres (NS), lecithin dispersion (LD), Myritol 318 dispersion (MD) and Pluronic F-68 solution (PS) $(0.5 \% \mathrm{w} / \mathrm{v})$. Each tested preparation had the same concentration of the different excipients present in xanthone-loaded nanocapsules, i.e. theoretical xanthone concentration equivalent. As shown in Figure 4 all formulations containing lecithin, such as empty nanocapsules, NE and LD, were associated with a reduction of NO production. Formulations without lecithin produced no reduction on NO levels. These results indicated that lecithin could be the excipient responsible for the inhibitory activity exhibited by empty nanocapsules. As it was previously reported by Aramaki et al. (1996), negatively charged unloaded liposomes containing phosphatidylserine or phosphatidic acid also elicited an inhibitory effect on NO production by macrophages as a result of suppression of NOS induction. In the present work, soybean lecithin used for nanocapsule preparation contained approximately $40 \%$ of phosphatidylcholine and other components, such as phosphatidic acid and phosphatidylserine, which might explain the inhibitory activity exhibited by empty nanocapsules.

The improvement of the inhibitory effect of both xanthones on NO production through nanoencapsulation could be due to an enhancement of the intracellular delivery of the compounds, as it was previously reported for different drugs (Morin et al. 1994, Wang and Zhang 2001). It is well known that the uptake of nanoparticles by macrophages occurs mainly by phagocytosis, which affords a specific targeting of drugs and immunomodulators to this type of cells (Maaßen et al. 1993, Legrand et al. 1999). Thus, phagocytosis of nanocapsules followed by release of the compounds in the lysosomes, as described by Seyler et al. (1999) for nanocapsules containing a derivative of muramyldipeptide, might be the main mechanism by which the intracellular delivery of xanthone and 3-methoxyxanthone was promoted. Furthermore, xanthones might also be transferred by diffusion after adsorption of nanocapsules onto the cells, or be released into the medium in the vicinity of cell membrane, generating a local concentration gradient that would favour the diffusion into the cells, as it was described by Maaßen et al. (1993) and Seyler et al. (1999).

\section{Uptake of nanocapsules by $\mathfrak{7 7 7 4}$ macrophage cell line}

In order to confirm the occurrence of a phagocytic process in the internalization of the nanocapsules a fluorescence microscopy technique was adopted and performed at different focus positions that allows identification more clearly if the observed fluorescence is located inside or outside the cells. Microphotographs of cells incubated at $37^{\circ} \mathrm{C}$ (Figure $5 \mathrm{a} 1$ and b1) showed

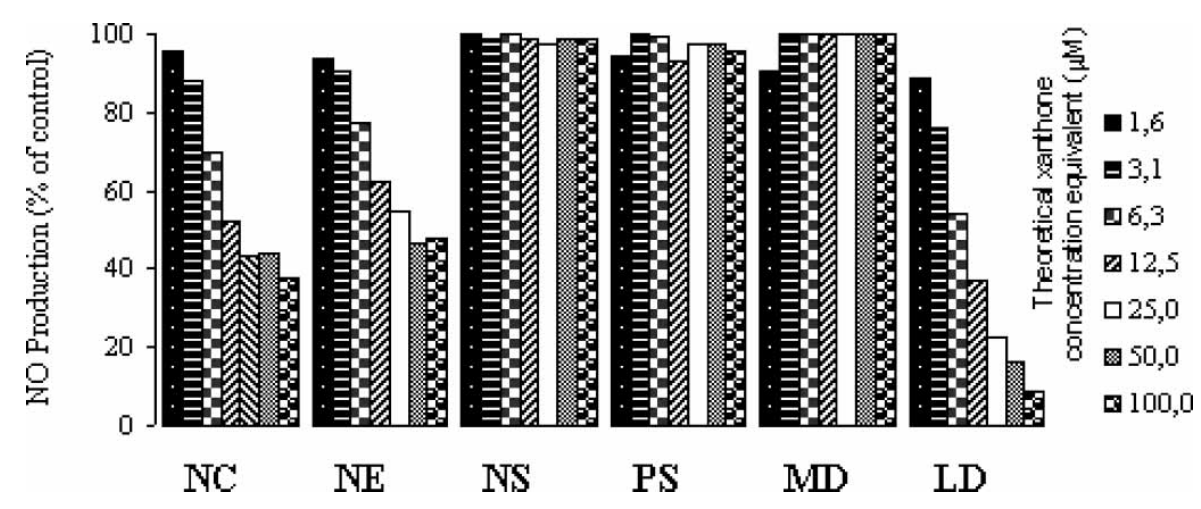

Figure 4. Effect of different formulations without xanthones on NO production by IFN- $\gamma /$ LPS stimulated J774 macrophages: Nanocapsules (NC), nanoemulsion (NE), nanospheres (NS), Pluronic F-68 solution (PS), Myritol 318 dispersion (MD) and lecithin dispersion (LD). Results show the mean values of three replicates from one representative experiment of two carried out independently. 

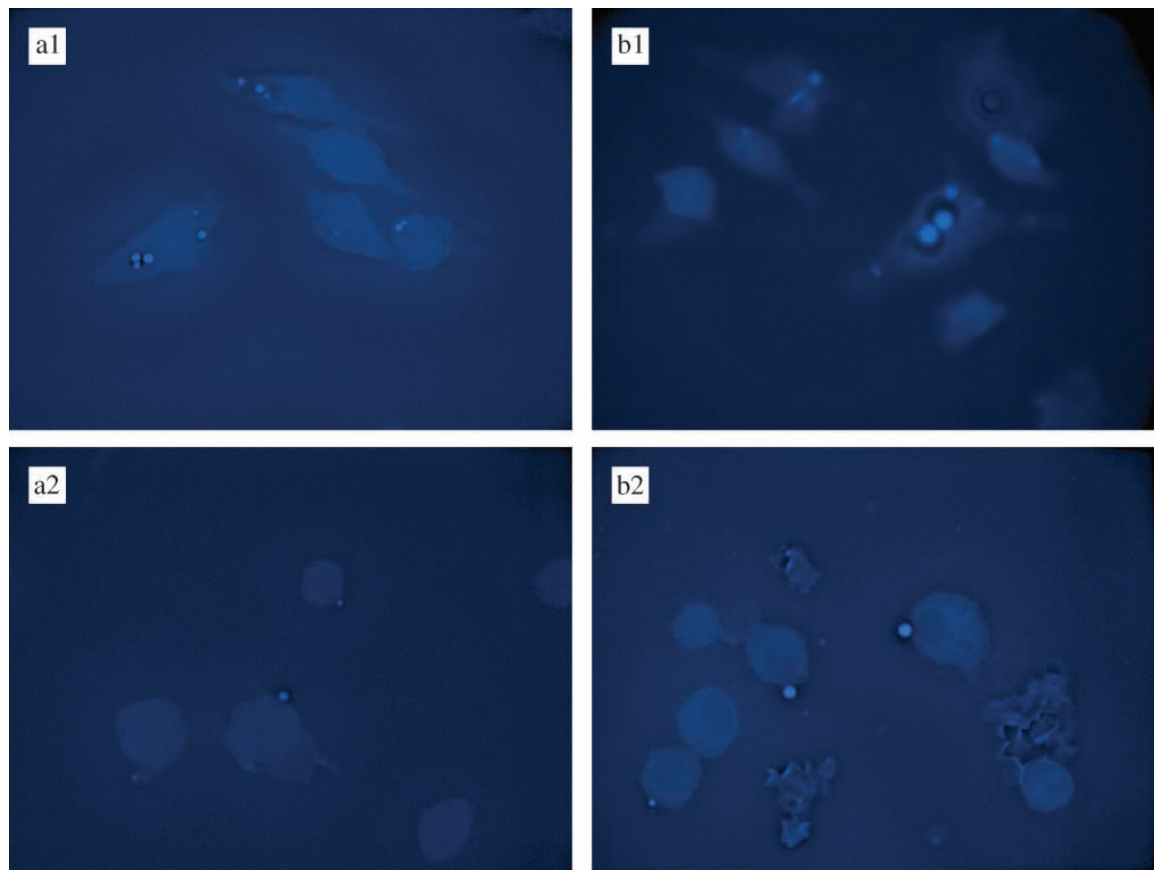

Figure 5. Fluorescent microphotographs of murine macrophage cell line J774 incubated with xanthone nanocapsules (a), and 3-methoxyxanthone nanocapsules (b) at $37^{\circ} \mathrm{C}(\mathrm{a} 1, \mathrm{~b} 1)$ and $4^{\circ} \mathrm{C}(\mathrm{a} 2, \mathrm{~b} 2)$ for $4 \mathrm{~h}$.

intramacrophage blue fluorescence concentrated in vesicular compartments, corresponding to xanthoneor 3-methoxyxanthone-loaded nanocapsules. Incubation at $4{ }^{\circ} \mathrm{C}$ resulted in a fluorescence located outside cells near macrophages surface (Figure $5 \mathrm{a} 2$ and b2). No fluorescence was observed either inside or outside the macrophages incubated with empty nanocapsules (results not shown). It is well known that the ability of macrophages to phagocyte particles is greatly suppressed at $4^{\circ} \mathrm{C}$ (Tabata and Ikada 1988). Thus, our results indicate that nanocapsule internalization, observed at $37^{\circ} \mathrm{C}$, involves a phagocytic process, whereas at $4^{\circ} \mathrm{C}$ a passive binding of nanocapsules to macrophage surface may occur. These results are in accordance with those reported by Mosqueira et al. (2001) for PLA nanocapsules containing a fluorescent probe, using the same J774 macrophage cell line.

\section{Conclusions}

A weak inhibitory effect on NO production by LPS/INF- $\gamma$ activated J774 macrophage cell line was observed for xanthone, even at the highest studied concentration $(100 \mu \mathrm{M})$. This effect was slightly higher for 3-methoxyxanthone. The encapsulation of these compounds in PLGA nanocapsules afforded a remarkable increase of the inhibitory effect on NO production. For xanthone-loaded nanocapsules a decrease of about $90 \%$ of NO production was achieved for $100 \mu \mathrm{M}$ concentration, whereas a reduction of
$80 \%$ was observed for an equal concentration of 3methoxyxanthone-loaded nanocapsules.

Fluorescence microphotographs confirmed that a phagocytic process was involved in the uptake of xanthone- and 3-methoxyxanthone-loaded PLGA nanocapsules by macrophages. Phagocytosis might be the main responsible mechanism for the enhancement of the intracellular delivery of both xanthones and consequently for the improvement of their biological effect.

Although the mechanism by which xanthone and 3-methoxyxanthone inhibits NO production by activated macrophages is not yet elucidated, the results presented here suggest that nanocapsule formulations of these xanthones are promising systems that might be used in disorders associated with production of high amounts of $\mathrm{NO}$ by macrophages. Moreover, these results are very encouraging for further studies concerning the improvement of other biological activities of xanthone and its derivatives through their incorporation in nanoparticles.

\section{Acknowledgements}

This work was supported by Fundação para a Ciência e a Tecnologia (FCT) (Unidade de I\&D no 226/94), POCTI (QCA III), FEDER and Praxis XXI. Fátima Cerqueira and Maribel Teixeira are recipients of $\mathrm{PhD}$ grants from FCT (Praxis XXI/BD/21801/99 and Praxis XXI/BD/21841/99). 


\section{References}

Aramaki Y. 2000. Liposomes as immunomodulator-inhibitory effect of liposomes on NO production from macrophages. Biol Pharm Bull 23:1267-1274.

Aramaki Y, Nitta F, Matsuno R, Morimura Y, Tsuchiya S. 1996. Inhibitory effects of negatively charged liposomes on nitric oxide production from macrophages stimulated by LPS. Biochem Biophys Res Commun 220:1-6.

De las Heras B, Navarro A, Godoy A, Villar AM. 1997. Inhibition of the induction of nitric oxide synthase in J774 macrophages by andalusol, a diterpenoid from Sideritis foetens clem. Pharm Pharmacol Lett 7:111-112.

Fernandes EGR, Silva AMS, Cavaleiro JAS, Silva FM, Borges MF, Pinto MMM. 1995. Hepatoprotective activity of xanthones and xanthonolignoids against tert-butylhydroperoxide-induced toxicity in isolated rat hepatocytes comparison with silybin. Pharm Res 12:1756-1760.

Fernandes EGR, Silva AMS, Cavaleiro JAS, Silva FM, Borges MF, Pinto MMM. 1998. ${ }^{1} \mathrm{H}$ and ${ }^{13} \mathrm{C}$ Spectroscopy of mono-, di-, tri- and tetrasubstituted xanthones. Magn Reson Chem 36:305-309.

García D, Delgado R, Ubeira FM, Leiro J. 2002. Modulation of rat macrophage function by the Mangifera indica L extracts Vimang and mangiferin. Int Immunopharmacol 2:707-806.

Gonzalez MJ, Nascimento MSJ, Cidade H, Pinto MM, Kijjoa A, Anantachoke C, Silva AMS, Herz W. 1999. Immunomodulatory activity of xanthones from Calophyllum teysmannii var. inuphylloide. Planta Méd 65:368-371.

Green BN, Warguer DA, Glowgoski J, Skipper PL, Wishnok JS, Tannenbaum JR. 1982. Analysis of nitrate, nitrite and (15) N nitrate in biological fluids. Anal Biochem 126:131-138.

Gross SS, Wollin MS. 1995. Nitric oxide: Pathophysiological mechanisms. Ann Rev Physiol 57:737-769.

Jain R, Shah NH, Malick AW, Rhodes C. 1998. Controlled drug delivery by biodegradable poly(ester) devices: Different preparative approaches. Drug Dev Ind Pharm 24:703-727.

Kolb H, Kolb-Bachofen V. 1992. Nitric oxide: A pathogenic factor in autoimmunity. Immunol Today 13:157-159.

Kuo PC, Schoeder RA. 1995. The emerging multifaceted roles of nitric oxide. Ann Surg 221:220-235.

Leiro JM, Álvarez E, Arranz JA, Siso IG, Orallo F. 2003. In vitro effects of mangiferin on superoxide concentrations and expression of the inducible nitric oxide synthase, tumour necrosis factor- $\alpha$ and transforming growth factor- $\beta$ genes. Biochem Pharmacol 65:1361-1371.

Lin CN, Chung MI, Liou SJ, Lee TH, Wang JP. 1996. Synthesis and anti-inflammatory effects of xanthone derivatives. J Pharm Pharmacol 48:532-538.

Lowenstein CJ, Alley EW, Raval P, Snowman AM, Snyder SH, Russell SW, Murphy WJ. 1993. Macrophage nitric oxide synthase gene: Two upstream regions mediate induction by interferon $\mathrm{g}$ and lipopolysaccharide. Proc Natl Acad Sci USA 90:9730-9734.

Maaßen S, Fattal F, Müller RH, Couvreur P. 1993. Cell cultures for the assessment of toxicity and uptake of polymeric particulate drug carriers. STP Pharma Sci 3:11-22.
Morin C, Barrat G, Fessi H, Devissaguet J-P, Puisieux F. 1994. Improved intracellular delivery of muramyl dipeptide analog by means of nanocapsules. Int $\mathrm{J}$ Immunopharmacol $16: 451-456$.

Mosqueira VC, Legrand P, Gulik A, Bourdon O, Gref R, Labarre D, Barratt G. 2001. Relationship between complement activation, cellular uptake and surface physicochemical aspects of novel PEG-modified nanocapsules. Biomaterials 22:2967-2979.

Mossman T. 1983. Rapid colorimetric assay for cellular growth and survival: Application to proliferation and cytotoxicity assays. J Immunol Methods 65:55-63.

Pedro M, Cerqueira F, Sousa ME, Nascimento MSJ, Pinto M. 2002. Xanthones as inhibitors of growth of human cancer cell lines and their effects on the proliferation of human lymphocytes in vitro. Bioorg Med Chem 10:3725-3730.

Peres V, Nagem TJ, de Oliveira FF. 2000. Tetraoxygenated naturally occurring xanthones. Phytochemistry 55:683-710.

Pinto M, Nascimento MSJ. 1997. Anticomplementary activity of hydroxy- and methoxyxanthones. Pharm Pharmacol Lett $7: 125-127$.

Pinto MMM, Sousa EP. 2003. Natural and synthetic xanthonolignoids: Chemistry and biological activities. Current Med Chemistry 10:1-12.

Saraiva L, Fresco P, Pinto E, Kijjoa A, Gonzalez MJ, Gonçalves J. 2002a. Differential activation of protein kinase C isoforms by euxanthone, revealed by an in vivo yeast phenotypic assay. Planta Med 68:1039-1041.

Saraiva L, Fresco P, Pinto E, Sousa E, Pinto M, Gonçalves J. 2002b. Synthesis and in vivo modulatory activity of protein kinase C of xanthone derivatives. Bioorg Med Chem 10:3219-3227.

Saraiva L, Fresco P, Pinto E, Sousa E, Pinto M, Gonçalves J. 2003. Inhibition of $\alpha, \beta I, \delta$ and $\zeta$ protein kinase $C$ isoforms by xanthonolignoids. J Enzyme Inhib Med Chem 18:357-370.

Seyler I, Appel M, Devissaguet J-P, Legrand P, Barratt G. 1999. Macrophage activation by a lipophilic derivative of muramyldipeptide within nanocapsules: Investigation of the mechanism of drug delivery. J Nanopart Res 1:91-97.

Tabata Y, Ihada Y. 1988. Macrophage phagocytosis of biodegradable microspheres composed of L-lactic acid/glycolic acid homoand copolymers. J Biomed Mater Res 22:837-858.

Teixeira M, Afonso CMM, Pinto MMM, Barbosa CM. 2003. A validated HPLC method for assay of xanthone and 3-methoxyxanthone in PLGA nanocapsules. J Chromatogr Sci 41:371-376.

Teixeira M, Alonso MJ, Pinto MMM, Barbosa CM. 2004. Development and characterization of PLGA nanospheres and nanocapsules containing xanthone and 3-methoxyxanthone. Eur J Pharm Biopharm (accepted for publication).

Thull U, Kneubuhler S, Testa B, Borges MFM, Pinto MM. 1993. Substituted xanthones as selective and reversible monoamine oxidase A (MAO-A) inhibitors. Pharm Res 10:1187-1190.

Wang J, Zhang Q. 2001. Uptake of cyclosporine A loaded colloidal drug carriers by mouse peritoneal macrophages in vitro. Acta Pharmacol Sin 22:57-61. 